\title{
STUDY OF THE ELECTROMAGENTIC CROSSTALK INTERFERENCE IN A PRINTED CIRCUIT BOARD BY COMPUTATIONAL METHODS
}

\author{
Elaine Santos ${ }^{1}$, Ailton Akira Shinoda ${ }^{2}$ \\ ${ }^{1}$ Faculdade de Engenharia de Ilha Solteira, Universidade Estadual Paulista, Ilha Solteira \\ (eng_elaine2004@yahoo.com.br)
}

${ }^{2}$ Faculdade de Engenharia de Ilha Solteira, Universidade Estadual Paulista, Ilha Solteira

\begin{abstract}
With increasing speeds of compression and digital signal ("clock") of electronic products, there is a growing concern with the service standards of EMI / EMC so that such products will be successful in certification testing of regulators such as the FCC (Federal Communications Commission) and Anatel (National Telecommunications Agency).

To this must have emission characteristics of electromagnetic interference and susceptibility to the appropriate type of equipment to which products belong, as in a printed circuit board (PCB). In equipment design, one important issue is compliance with relevant EMC standards. Effects such as crosstalk, reflections on transmission lines, impulse noise as the simultaneous transition of levels, electrostatic discharge, current spike and radiation or electromagnetic radiation should be considered when using high-speed integrated circuits. These effects combined with the nonlinear characteristics of passive components can cause an erroneous operation at the component level, subsystem and system.

Crosstalk is one of the four families of signal-integrity problems. In the simplest terms, crosstalk is unwanted coupling of voltages and currents between neighboring conductors. On a PCB, the conductors are usually traces, although crosstalk can also occur in connectors, cables, and component packages. In the classic scenario, when a signal is intentionally driven on one conductor, an unwanted signal also appears on a neighboring conductor-even though there is no conductive connection between the driven conductor and its neighbor. The noise margin is typically about $15 \%$ of the total single-voltage swing, but varies among device families. Of this, about $1 / 3$ or $5 \%$ of the signal swing, is typically allocated to crosstalk. Unfortunately, the magnitude of the noise generated in typical traces on board can often be larger than 5\%. This is why it is important to be able to predict the magnitude of crosstalk, indentify the origin of excessive noise, and actively work to minimize the crosstalk in the design of packages, connectors, and board-level interconnects. Understanding the origin of the problem and how to design interconnects with reduced crosstalk is increasingly important as rise time decreases.

It is important during the development phase of a project to have tools or techniques that can assist with possible problems that may appear only when the equipment is in the field. The costs of re-design and new manufacturing processes can be very high. Therefore, the use of computerized simulation tools is extremely important.
\end{abstract}

Keywords: Electromagnetic, Crosstalk, PCB. 


\section{INTRODUCTION}

With increasing speeds of compression and digital signal ("clock") of electronic products, there is a growing concern with the service standards of EMI / EMC so that such products will be successful in certification testing of regulators such as the FCC (Federal Communications Commission) and Anatel (National Telecommunications Agency) [5].

To this must have emission characteristics of electromagnetic interference and susceptibility to the appropriate type of equipment to which products belong, as in a printed circuit board (PCB). In equipment design, one important issue is compliance with relevant EMC standards. Effects such as crosstalk, reflections on transmission lines, impulse noise as the simultaneous transition of levels, electrostatic discharge, current spike and radiation or electromagnetic radiation should be considered when using high-speed integrated circuits [6]. These effects combined with the nonlinear characteristics of passive components can cause an erroneous operation at the component level, subsystem and system.

To this must have emission characteristics of electromagnetic interference and susceptibility to the appropriate type of equipment to which products belong, as in a PCB. In equipment design, one important issue is compliance with relevant EMC standards [6]. Effects such as crosstalk, reflections on transmission lines, impulse noise as the simultaneous transition of levels, electrostatic discharge, current spike and radiation or electromagnetic radiation should be considered when using high-speed integrated circuits [4].

Crosstalk is one of the four families of signal-integrity problems. In the simplest terms, crosstalk is unwanted coupling of voltages and currents between neighboring conductors. On a PCB, the conductors are usually traces, although crosstalk can also occur in connectors, cables, and component packages. In the classic scenario, when a signal is intentionally driven on one conductor, an unwanted signal also appears on a neighboring conductor -even though there is no conductive connection between the driven conductor and its neighbor. The noise margin is typically about $15 \%$ of the total single-voltage swing, but varies among device families. Of this, about $1 / 3$ or $5 \%$ of the signal swing is typically allocated to crosstalk [1]. Unfortunately, the magnitude of the noise generated in typical traces on board can often be larger than $5 \%$. This is why it is important to be able to predict the magnitude of crosstalk, indentify the origin of excessive noise, and actively work to minimize the crosstalk in the design of packages, connectors, and board-level interconnects. Understanding the origin of the problem and how to design interconnects with reduced crosstalk is increasingly important as rise time decreases.

It is important during the development phase of a project to have tools or techniques that can assist with possible problems that may appear only when the equipment is in the field. The costs of re-design and new manufacturing processes can be very high. Therefore, the use of computerized simulation tools is extremely important.

To illustrate the useful of computational methods tool this article presents a case study of use of computer simulation to anticipate the behavior of operation taking into account the crosstalk effects and how to minimize the problems caused by this kind of noise.

The work is organized in the following order. Section 2 explains how the crosstalk is generated in PCB. Section 3 describes the circuit or net used as an example from PCB. Section 4 shows the effects of crosstalk in signal integrity.

\section{CROSSTALK}

In few words, crosstalk is unwanted coupling of voltages and currents between neigh- 
boring conductors. On a PCB, the conductors are generally traces, although crosstalk can also occur in connectors, cables, and component packages. In the usual crosstalk scenario, when a signal is intentionally driven on one conductor, an unwanted signal also appears on a neighboring conductor - even though there is no conductive connection between the driven conductor and its neighbor [2].

In a crosstalk scenario, any trace that is intentionally driven (usually by a switching IC output buffer) and is therefore a potential source of crosstalk on other traces is called an aggressor net. Any trace that potentially receives unwanted crosstalk from an aggressor net is called a victim net.

When a signal travels down a trace, it is an electromagnetic wave that is propagating along the trace, from the driver end toward the trace's far end. At points along the trace which the wave has already reached, transient voltages appear and currents flow, in response to the waves's presence.

Electromagnetic waves consist of time-changing electrical and magnetic fields. The fields are not confined to the inside of the trace that carries then - in fact, just the opposite: the fields' energy exists very predominantly outside the trace.

When a signal propagates down a transmission lines, there are electric-field lines between the signal and return paths and rings of magnetic-field lines around the signal and return path conductors. These fields are not confined to the immediate space between the signal and return paths. Rather, they spread out into the surrounding volume. These fields that spread out are called fringe fields.

Of course, the fringe fields drop off very quickly as it moves farther away from the conductors. Figure 1 shows the fringe fields between a signal path and a return path.

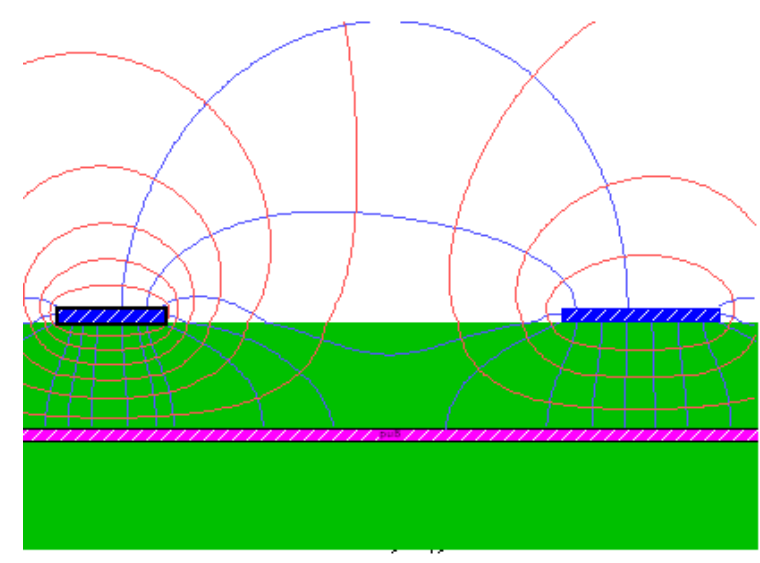

(a) far away

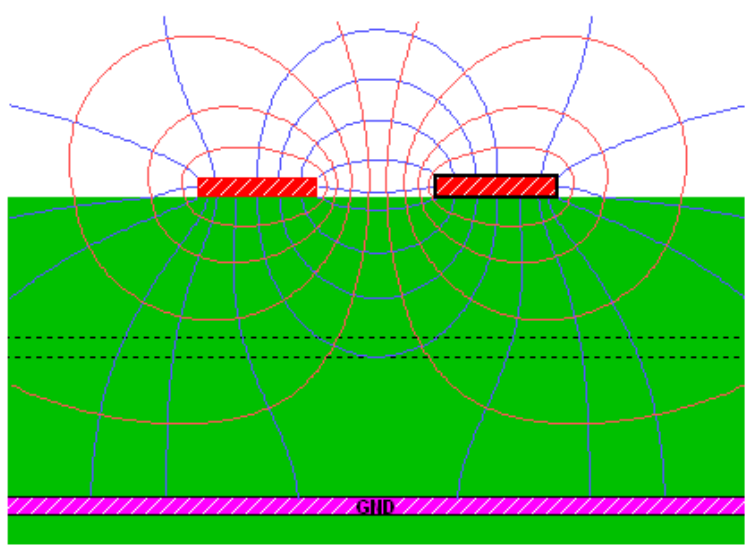

(b) near

Figure 1: Fringe fields near a signal line.

\section{CIRCUIT CONFIGURATION}

Figure 2 shows part of a telephone exchange circuit board that was developed. 


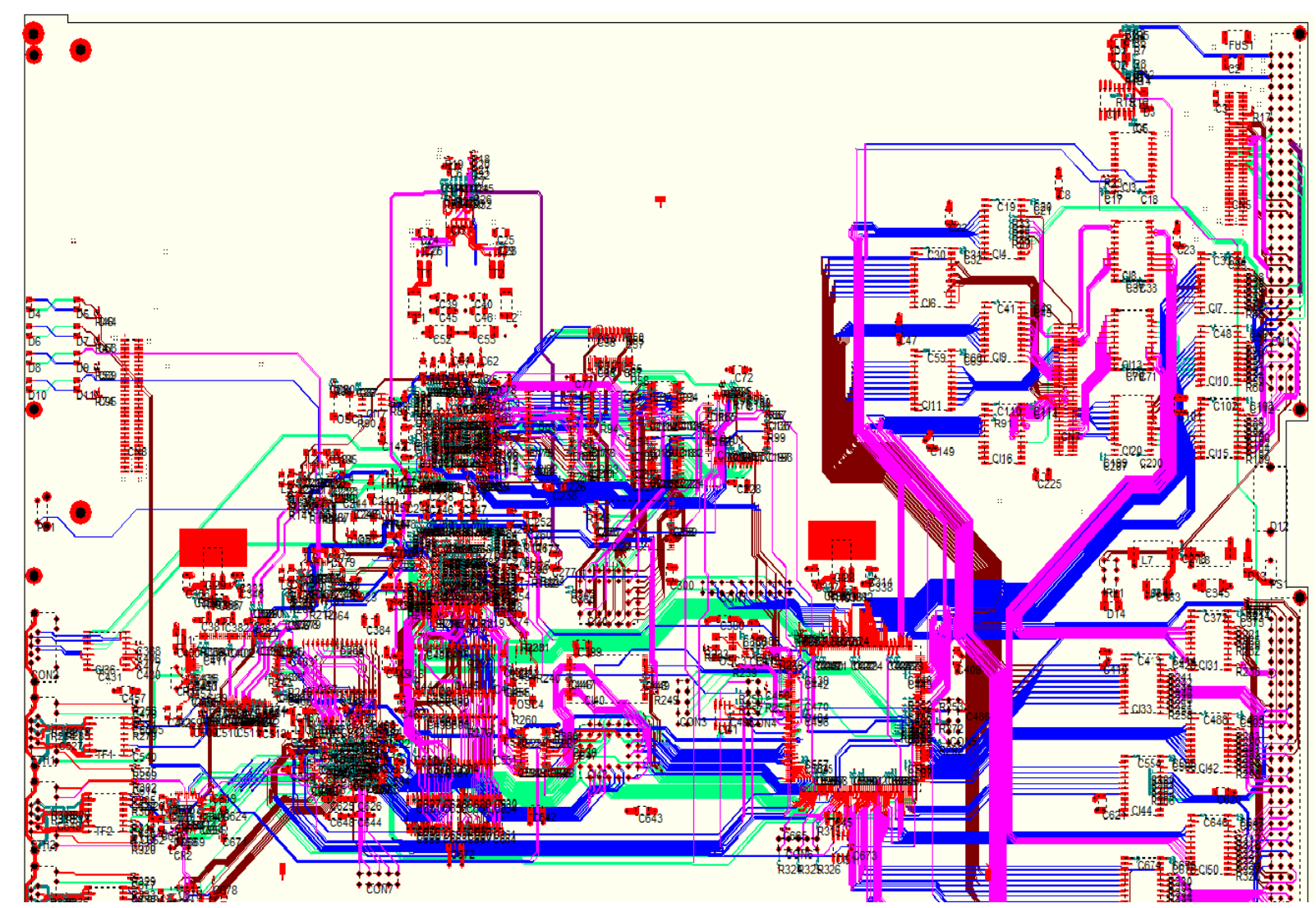

Figure 2: PCB.

Figure 3 shows the stackup employed in the board.

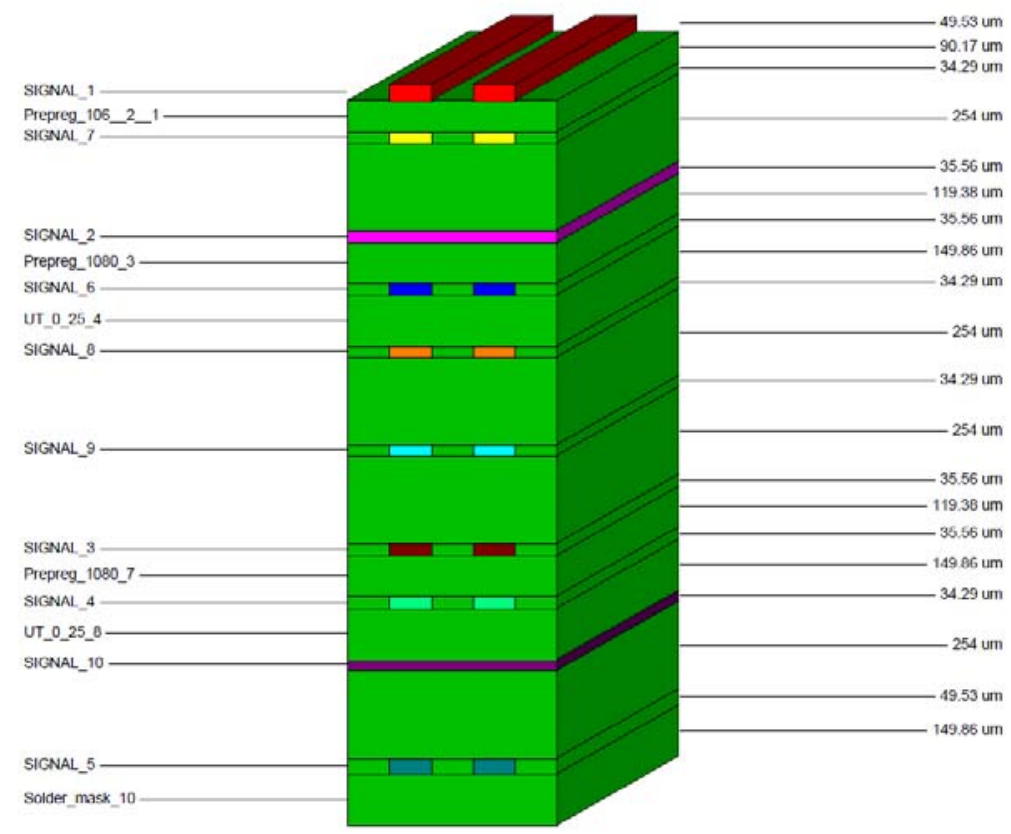

Figure 3: Stackup.

Figure 4 shows the layer board stackup in details. 


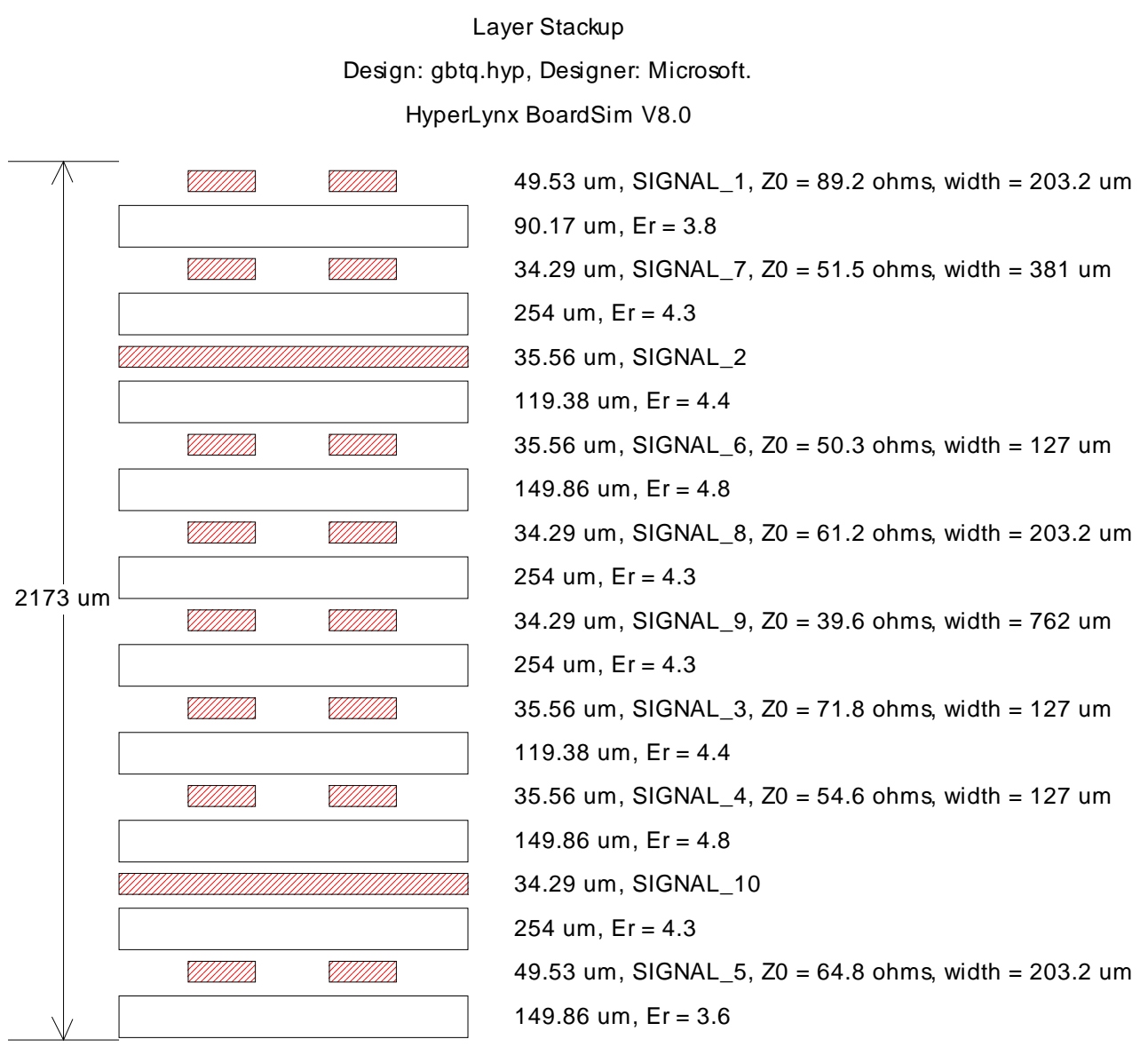

Figure 4: Layer Stackup.

\section{EFFECTS OF CROSSTALK IN SIGNAL INTEGRITY}

In order to verify the effects of crosstalk in signal integrity, HyperLynx Mentor Graphics ${ }^{\circledR}[3]$ software was used to simulate the subsystem circuit board that was developed for the telephone exchange. The aggressor net selected for simulation is illustrated in Figure 5.

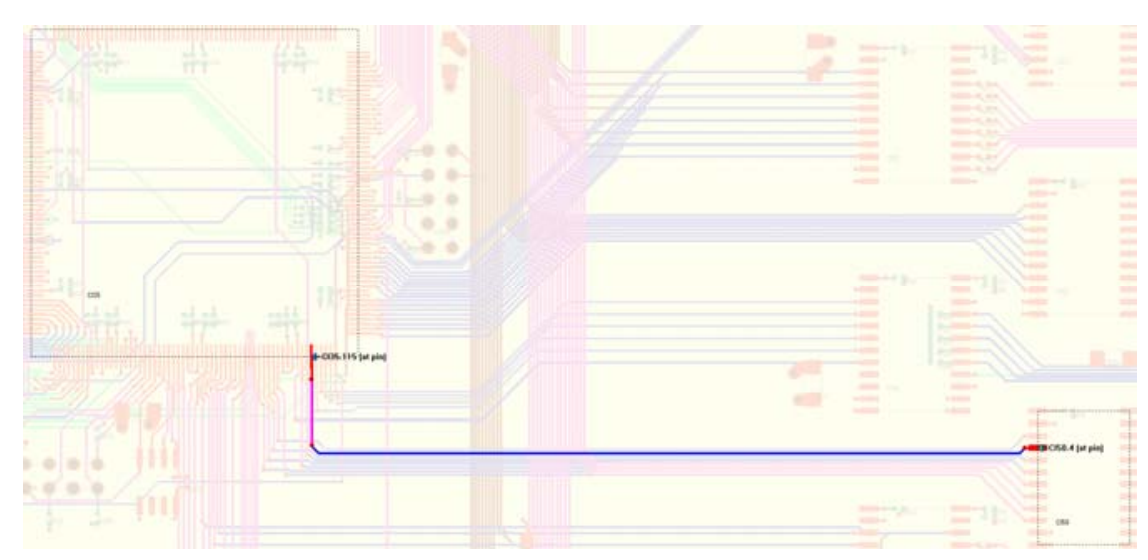

Figure 5: Aggressor net selected 
The block on the left is an FPGA device family from Altera ${ }^{\circledR C}$ CycloneIV connected to a CMOS device family 74LVCC in the right block.

Figure 6 shows the victim nets with its associated aggressor net (Figure 5).

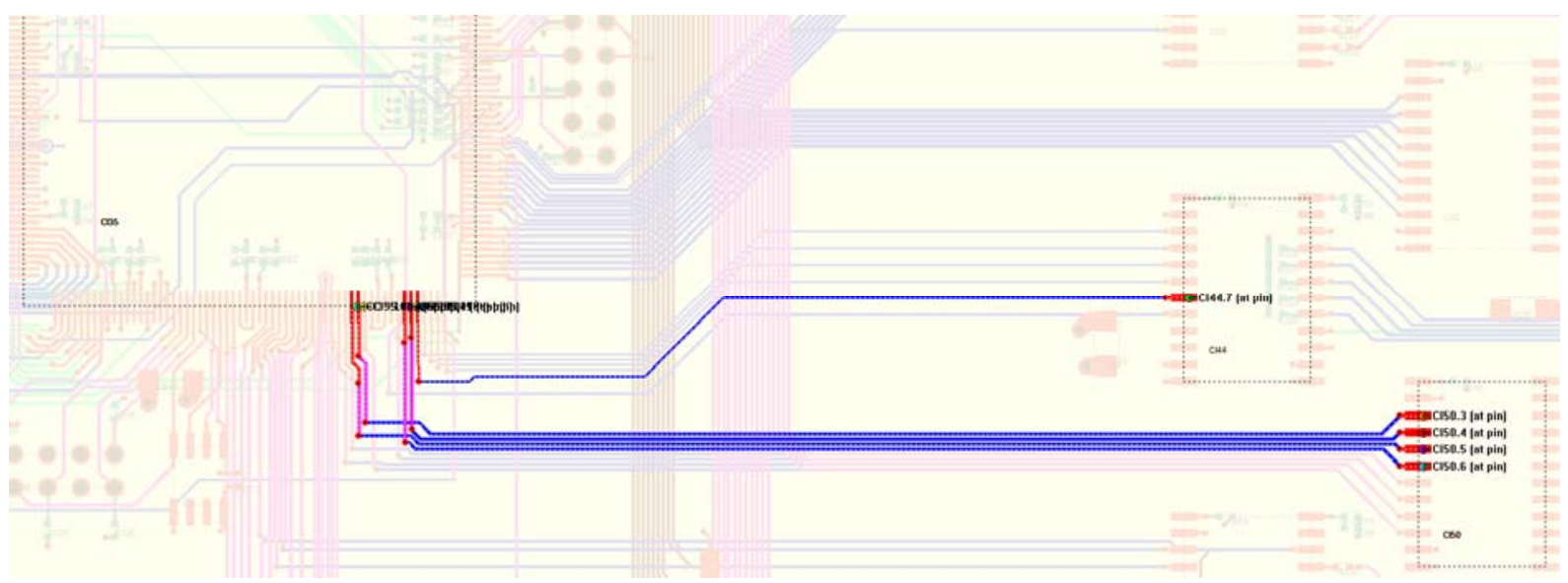

Figure 6: Victim nets

Figure 7 shows the electromagnetic field lines generated by the aggressor net responsible for the coupling of neighboring or victim nets.

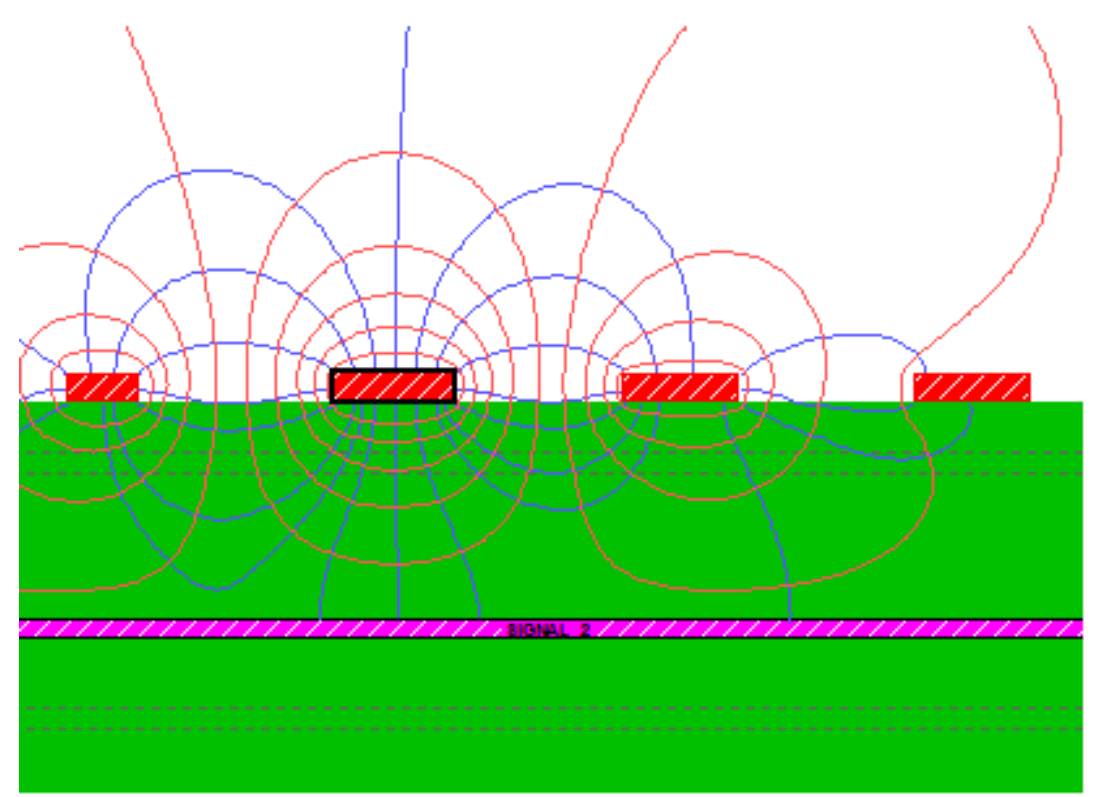

Figure 7: Electromagnetic field lines

Figure 8 shows the curve of the transition from high to low level generated by aggressor net. The fall time (10-90\%) was 0.544 ns for the devices used. 


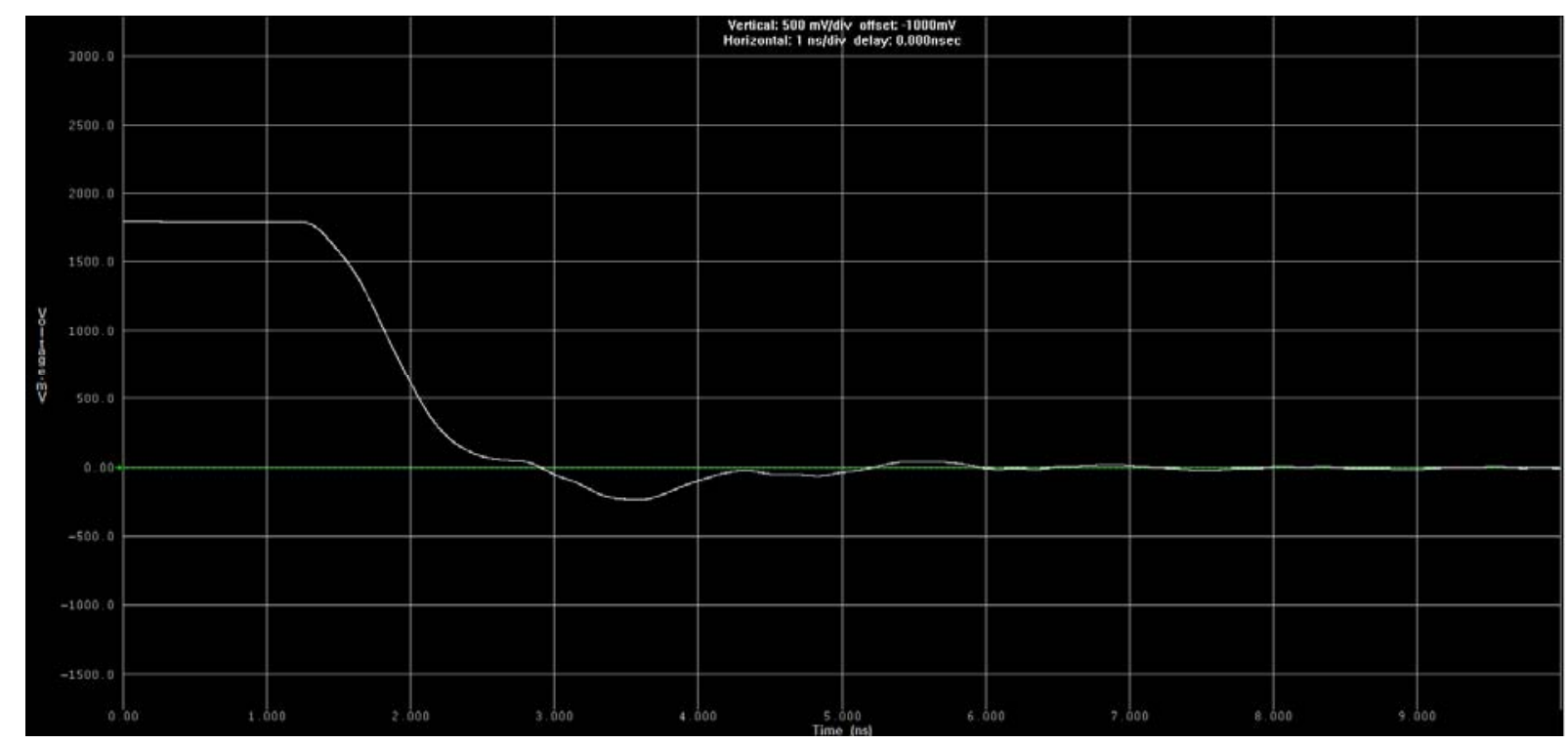

Figure 8: Transition level from aggressor net

Figure 9 shows the noise generated (crosstalk) in the victim nets due the change or transition from the aggressor net.

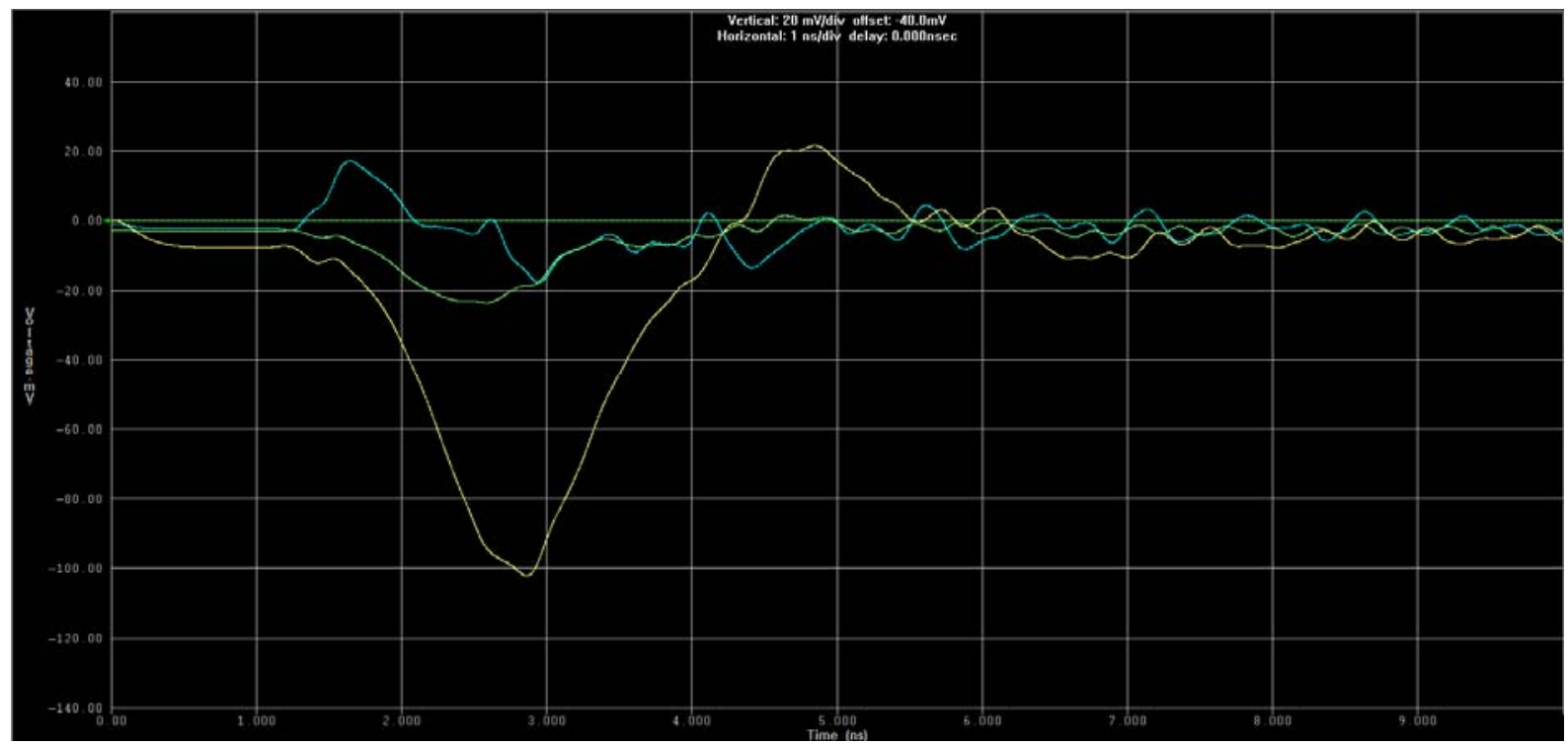

Figure 9: Crosstalk noise in the victim nets

\section{CONCLUSION}

Crosstalk noise and electromagnetic radiation in electronic systems are becoming serious problems with the increase in operating speed of digital circuits in PCB, i.e, the interconnect size becomes comparable to the wavelength of the exciting signal. This implies that the analysis of circuit should be made in terms of transmission line model.

It was analyzed a PCB employed in the telephone exchange equipment by HyperLynx Mentor Graphics ${ }^{\circledR}$ software, the results indicated that the worst magnitude of the noise generated due to crosstalk effect was $100 \mathrm{mV}$ (Figure 9) or 5.6\% from $1.8 \mathrm{~V}$ (Figure 8), in the limit of the margin. 


\section{Acknowledgements}

The authors would like to acknowledge the TROPICO Sistemas e Telecomunicações da Amazônia Ltda for funding this project.

\section{REFERENCES}

[1] Bogatin, E., Signal and Power Integrity, $2^{\text {nd }}$ Edition, Prentice Hall, 2010.

[2] Kaer, H., Wenyi, L. and Hongcheng, Y., The Signal Integrity of The High-speed IC Design, Frontier in Education Conference, 2009. FIE'09.39 ${ }^{\text {th }}$ IEEE.

[3] Mentor Graphics, Boardsim User Guide, 2006.

[4] Ott, H.W, Electromagnetic Compatibility Engineering, 1st Edition, John Wiley, 2009.

[5] Paul, C. R., Introduction to Electromagnetic Compatibility, 2nd Edition, John Wiley \& Sons, 2006.

[6] Rao, N. et al, EMC analysis in PCB design using an expert system, International Conference on Electromagnetic Interference and Compatibility, pp. 59-62, 1995. 